Article

\title{
Wavelength Scaling of Laser Wakefield Acceleration for the EuPRAXIA Design Point
}

\author{
Craig W. Siders, Thomas Galvin *, Alvin Erlandson, Andrew Bayramian, Brendan Reagan, \\ Emily Sistrunk, Thomas Spinka and Constantin Haefner
}

Lawrence Livermore National Laboratory, 7000 East Avenue, Livermore, CA 94551, USA

* Correspondence: galvin7@llnl.gov

Received: 1 June 2019; Accepted: 17 August 2019; Published: 21 August 2019

\begin{abstract}
Scaling the particle beam luminosity from laser wakefield accelerators to meet the needs of the physics community requires a significant, thousand-fold increase in the average power of the driving lasers. Multipulse extraction is a promising technique capable of scaling high peak power lasers by that thousand-fold increase in average power. However, several of the best candidate materials for use in multipulse extraction amplifiers lase at wavelengths far from the $0.8-1.0 \mu \mathrm{m}$ region which currently dominates laser wakefield research. In particular, we have identified Tm:YLF, which lases near $1.9 \mu \mathrm{m}$, as the most promising candidate for high average power multipulse extraction amplifiers. Current schemes to scale the laser, plasma, and electron beam parameters to alternative wavelengths are unnecessarily restrictive in that they stress laser performance gains to keep plasma conditions constant. In this paper, we present a new and more general scheme for wavelength scaling a laser wakefield acceleration (LWFA) design point that provides greater flexibility in trading laser, plasma, and electron beam parameters within a particular design point. Finally, a multipulse extraction $1.9 \mu \mathrm{m}$ Tm:YLF laser design meeting the EuPRAXIA project's laser goals is discussed.
\end{abstract}

Keywords: laser wakefield accelerators; multipulse extraction; petawatt

\section{Introduction}

Laser wakefield acceleration (LWFA) was invented by Tajima and Dawson [1] two decades before the critical 2018 Nobel Prize-winning innovation of Chirped Pulse Amplification (CPA) [2] enabled the construction of lasers of sufficient optical field strength to excite a laser wakefield. From the earliest wakefield observations (for example [3-7] and experiments summarized in Esarey's excellent review [8]), Titanium-doped sapphire (Ti:S) [3-5] as well as Nd:Glass [6,7], and more recently ytterbium-doped materials have been the laser materials of choice due to their large bandwidths and optimal saturation fluences which permit the production of the necessary $~ 100-\mathrm{TW}$ peak power robustly and without optical damage, and in compact university-scale facilities. The average power of ultrafast lasers experienced a $1000 \times$ increase as well, with the state-of-the-art milliwatt power levels of ultrafast dye lasers rapidly becoming watt-scale average power levels of Ti:S lasers. The quarter century dominance of Ti:S has seen a further 100× increase, with the current world-leading High-repetition-rate Advanced Petawatt Laser System (HAPLS) laser capable of operating at 300-W average power [9]. For Tajima and Dawson's invention to deliver on its potential of $\mathrm{TeV}$ laser-driven particle accelerators, a further $1000 \times$ increase in laser average power is necessary to provide luminosities on par with the needs of the particle physics community. Achieving this goal will require a top-level systems engineering approach within which the operating conditions of the drive lasers, underdense plasmas, and electron beams can be traded off against each other to achieve the highest overall performance of the laser-driven plasma accelerator and collider. This paper will present a new and more general scheme for wavelength scaling laser wakefield operating parameters, illustrate that schemes alternative 
to the current standard [10] have particular advantages, and introduce a new laser architecture and laser material, operating at a longer $2-\mu \mathrm{m}$ wavelength, which we ascertain to be capable of meeting the needed thousand-fold increase in average power. This motivates the need for the broader LWFA community to consider a broader design space which includes a new laser architecture and material and new laser wavelengths. We hope that, in this way, a route to realizing Tajima and Dawson's 1979 vision may be found.

Overall energy and power requirements are a good top-level systems engineering trade space to start from. Figure 1 shows plasma wakefield (both laser and particle beam) drivers utilized for existing (solid boundary) and planned (dotted boundary) systems, as well as regions of peak-power/ average-power useful for future applications in high-gradient accelerators and electron-beam pumped free-electron lasers (FELs), as detailed in the 2016 Department of Energy (DOE) Advanced Accelerator Development Strategy Report [11] and the EuPRAXIA (European Plasma Research Accelerator with eXcellence In Applications) project [12]. Also included in this chart are points for particle-beam driven schemes such as CERN's proton-based AWAKE [13] and SLAC's electron-based FACET [14] and FACET-II. For these, the bunch charge, duration, and repetition rates were used to estimate the peak power and average power in the charged particle beam. It is notable that the peak and average powers of both laser and particle beam sources are comparable in both their current states-of-the-art and also that both require $\sim 100 \times$ increase in average power needed to get to reach anticipated FEL, or $\sim 1000 \times$ increase in average power needed to reach the 5-10 GeV per stage design point frequently used in future TeV-class collider designs. It is also notable to compare the physical footprints of the beam-driven AWAKE and FACET-II systems, which are housed in major facility-scale buildings, to the room/laboratory sized lasers included on this diagram.

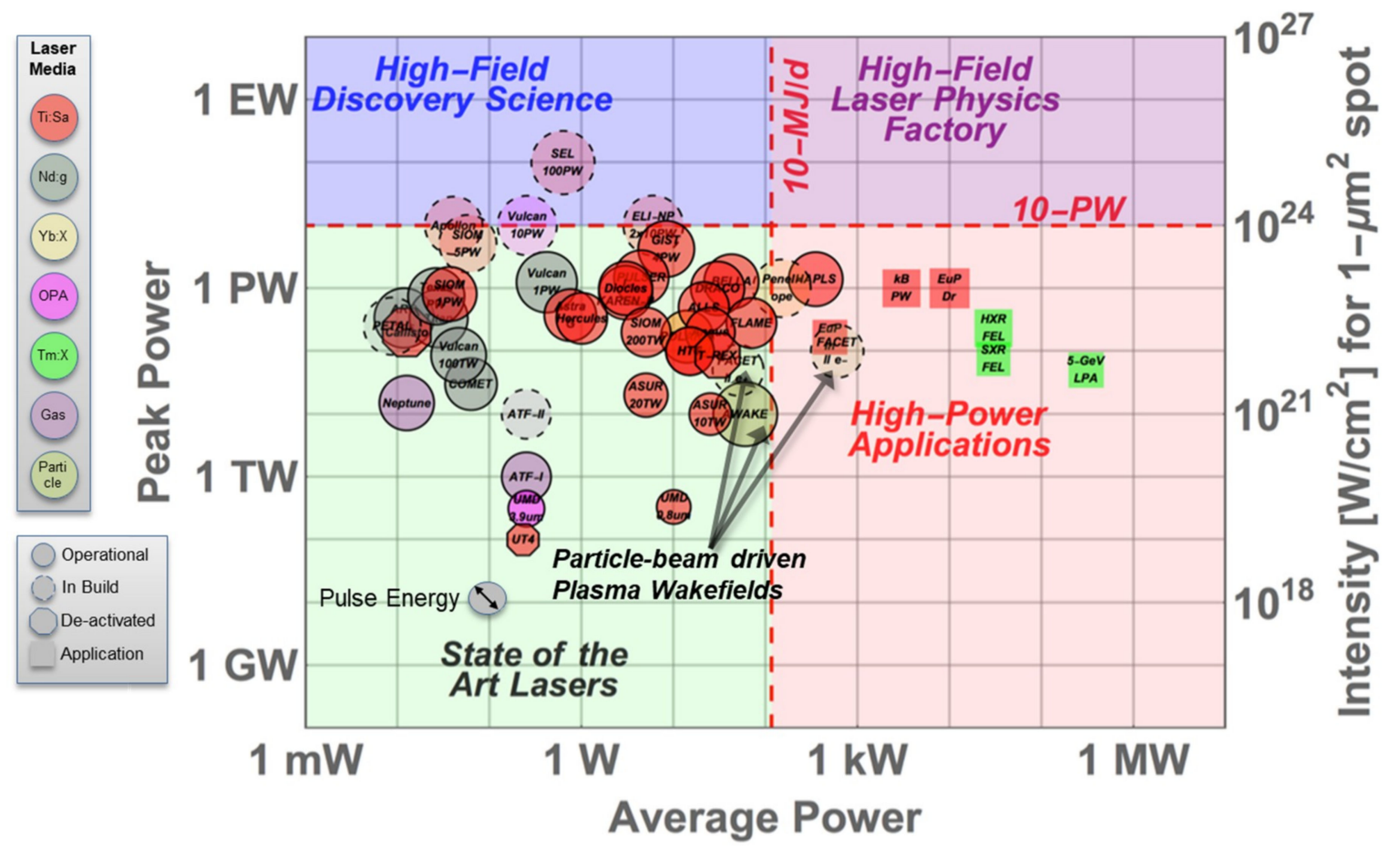

Figure 1. Current high-peak-power lasers and particle drivers for wakefield accelerator experiments, and some stated LWFA applications.

Increasing the repetition-rates and average powers of $0.1-1$ PW class lasers a thousand-fold is not as straightforward as simply pulsing current state-of-the-art laser architectures more frequently at the same pulse energy. The long-standing material of choice in high power lasers, Ti:S, has a 3.2- $\mu$ s upper state lifetime and so must itself be optically pumped by intense GW-scale high peak power lasers. The high quantum defect of Ti:S (34\%) has two detrimental consequences: first, that approximately a 
third of the absorbed pump light is converted to waste heat in the crystal; and second, that the pump laser must be shifted in wavelength to the green. The second-harmonic generation of high-energy, ns-scale 1- $\mu \mathrm{m}$ laser pulses are thus the pump-laser of choice for Ti:S. The current world-leading HAPLS laser [9] is an excellent example of using diode-pumped Nd:glass to provide multi-kW of 1- $\mu \mathrm{m}$ light for effective doubling and pumping of Ti:S. Gas-flow cooling of both the multi-slab Nd:glass and multi-slab Ti:S media in HAPLS is crucial for removing the considerable waste heat generated, given the net few-percent wall-plug efficiency theoretically obtainable with this DPSSL (diode-pumped solid-state laser)-pumped Ti:S architecture ( 10× greater than with flash-lamp pumping). We estimate that Ti:S architectures will not efficiently scale beyond a few-kHz rep-rate at the energies and peak powers needed for future accelerators.

A first step towards greater net efficiencies as well as higher pulse energies is to utilize direct diode-pumped chirped pulse amplification in the Nd:glass itself-a configuration we have termed SHARC (Scalable High-power Advanced Radiographic Capability). SHARC is a hybrid between the HAPLS pump laser and the NIF ARC laser, which is a $\mathrm{kJ} /$ ps scale chirped-pulse amplification (CPA) laser based upon large-aperture Nd:glass. By eliminating the Ti:S stage and directly using $\mathrm{Nd}$ :Glass for short pulse amplification, a SHARC architecture can be $2-3 \times$ more efficient at the 150-J/150-fs/1-PW/10-Hz design point. Though burst-mode operation can increase the effective rep-rate to a few hundred micro-pulses per second, scaling this pulse-pumped architecture beyond the current state-of-art 10-Hz rapidly reaches thermal management and material shock limits.

Traditional high-energy short-pulse amplifiers are based on pulse-pumped, single pulse extraction (SPE), in which each shot represents a complete pump/extraction cycle where efficient operation requires nearly complete de-excitation of the stored energy via stimulated emission. We propose an alternative scheme, multipulse extraction (MPE), in which the gain medium is pumped continuously, and the upper state population is extracted over many pulses. MPE requires that the gain material have an inverse lifetime significantly less than the desired repetition rate. This method has three primary benefits: first, because efficient extraction is not necessary in a single pulse, the extraction fluence (and hence the B-integral) can be much lower than in an SPE design; second, there isn't a need to pump the gain medium within a single inverse lifetime, and therefore less expensive, less complex, and more efficient CW pump sources can be used; and finally, MPE enables the use of high-saturation-fluence materials. These materials may have important advantages, such as the ability to be directly diode-pumped, a lower quantum defect, or being in a more useful wavelength regime.

Due largely to its 15-ms gain lifetime, we have identified Tm:YLF as being an especially promising material for MPE high-average-power operation. Its operating wavelength of $1.9 \mu \mathrm{m}$, however, is far from the either $\sim 0.8-1 \mu \mathrm{m}$ or $10-\mu \mathrm{m}$ wavelengths at which LWFA has been demonstrated and explored. Existing schemes to scale the wavelengths of LWFA pump lasers, such as that presented by Schroeder [10], would suggest that utilizing longer drive laser wavelengths requires many more stages (by the square of wavelength) to achieve the same electron beam collision energies and luminosity. In this paper, Schroeder's wavelength scaling of multi-stage LWFAs will be briefly reviewed and a generalized method for wavelength scaling LWFA designs will be presented. Several instances of the new scaling method will be discussed, and a design for a multipulse extraction laser, operating at $1.9 \mu \mathrm{m}$ and meeting the requirements of the EuPRAXIA request for applications (RFA) [12], will then be discussed. Due to the large number of variables used in this paper, an alphabetized list of definitions is provided in Appendix A.

Based upon the considerations above, Figure 2 illustrates our current conclusions on the power scaling potential of Ti:S, Nd:Glass-based SHARC, Tm:YLF Big Aperture Thulium (BAT), and $\mathrm{CO}_{2}$ architectures. Luminosity is defined from $\mathcal{L}=f \frac{N^{2}}{4 \pi \sigma^{2}}$, where $f$ is the bunch rate, $N$ the number of electrons, and $\sigma^{2}$ the root mean square (RMS) electron bunch spot area. Due fundamentally to rep-rate limitations derived from thermal management considerations, we ascertain that Ti:S and SHARC, though adequate for near-term science studies and risk reduction activities, are not scalable to rep-rates needed for the targeted luminosity of a TeV-class collider. $\mathrm{CO}_{2}$, which arguably has considerable 
scaling potential via its long wavelength, has to date not been demonstrated with sufficiently short pulse durations (and cycles per pulse) nor has a scalable path to high average power at high peak power and efficiency been identified.

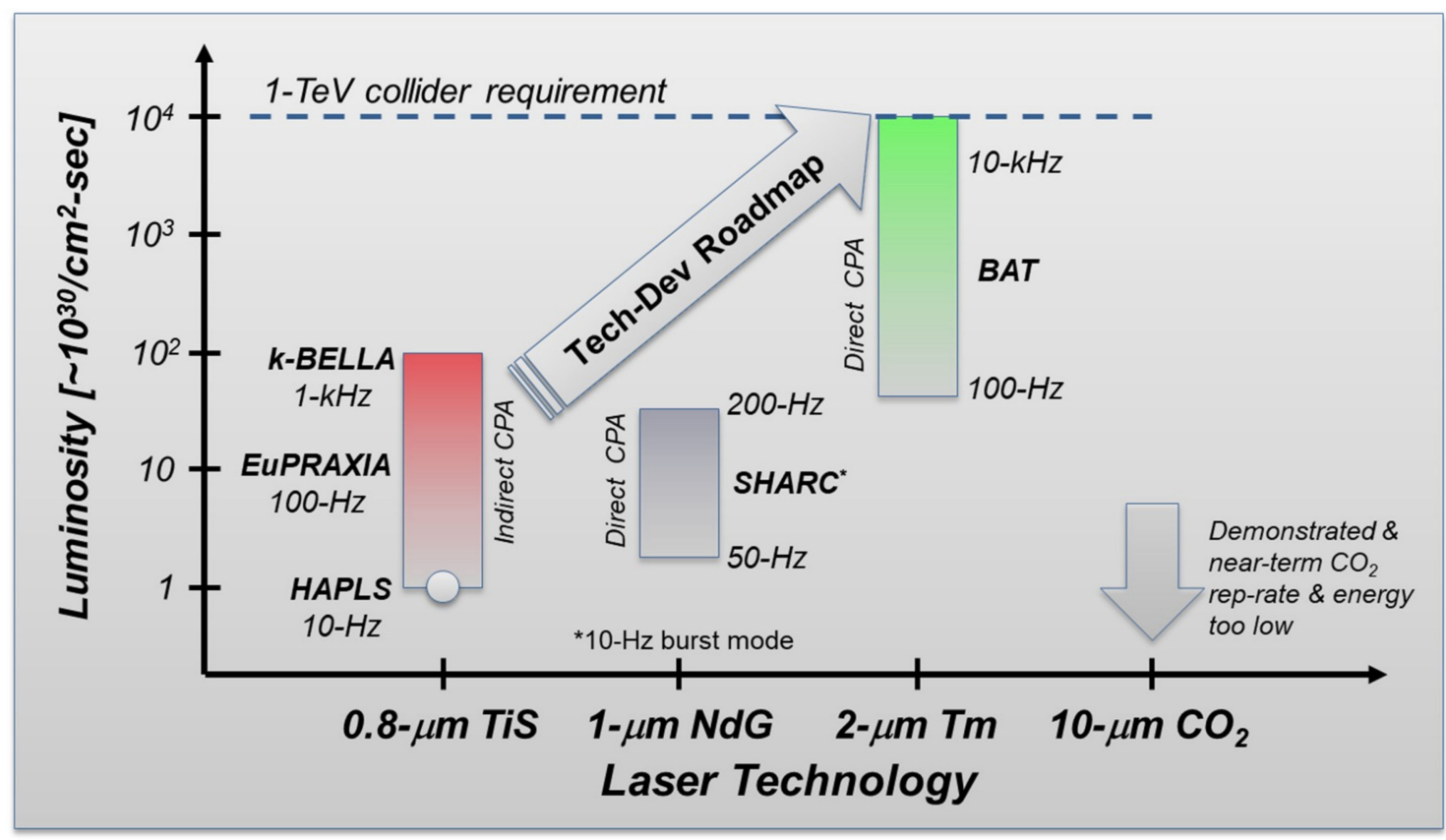

Figure 2. Collision luminosities scaling for current and proposed LWFA drive lasers. TiS and NdG (Nd:glass) lasers can be scaled in rep-rate to $\sim \mathrm{kHz}$ and $0.2-\mathrm{kHz}$, respectively, and so are at best near-term stepping stones towards the $10^{34} / \mathrm{cm}^{2}$-sec luminosity goal for a future TeV-scale collider. Multi-pulse extraction lasers based upon Tm:YLF (BAT) can scale to 10 's of $\mathrm{kHz}$ necessary to meet this luminosity.

\section{Wavelength Scaling}

In this section, we present the net scaling with wavelength for any given laser wakefield accelerator design point-that is, how a parameter depends on wavelength after including the wavelength scaling of any other variables in the typical engineering equations used for wakefield design and operation. A LWFA design point is determined by selecting the center of mass collision energy $\left(E_{c m}\right)$, the normalized vector potential of the laser field $\left(a_{0}\right)$, and efficiency $\left(\Gamma\right.$, which scales with $\left.a_{0}\right)$. Effective excitation of a wakefield using that $a_{0}$ and providing the desired $E_{c m}$ and $\Gamma$ requires satisfying the axial and transverse matching conditions $\omega_{p} \tau \sim f\left(a_{0}^{\zeta}\right) \sim$ constant, and $k_{p} D \sim f\left(a_{0}^{\phi}\right) \sim$ constant, which imposes a relationship between the density-dependent plasma frequency $\omega_{p}$ and laser pulse duration $\tau$, and between the plasma wavenumber $k_{p}=\frac{\omega_{p}}{c}$, and laser intensity full width at half maximum (FWHM) spot size $D$.

In Reference [10], Schroeder states that "for fixed $a_{0}$ and $k_{p} D$, the required peak laser power per LPA [laser plasma accelerator] stage scales as $P \propto n_{e}^{-1} \lambda^{-2}$ and the laser energy per LPA stage scales as $U_{L} \sim P \tau \propto n_{e}^{-\frac{3}{2}} \lambda^{-2}$ [variable names changed to agree with usage in this paper]." Schroeder assumes that electron density is held fixed in his scaling in order to maintain the same density and diameter of the guiding plasma channel. This is an unnecessarily restrictive imposition as that then implies that the laser spot size and pulse duration are fixed. For longer wavelengths, then, this greatly stresses the performance of the laser focusing and fractional bandwidth. A more general solution, which we will show admits to a more balanced system-level trade between plasma and laser parameters, is to scale wavelength under the constraint that $a_{0}^{2} \sim P\left(\frac{\lambda}{D}\right)^{2} \sim$ constant, is presented here. We do this by introducing a scaling parameter, $\eta$, and allowing the peak laser power scale as $P \sim \lambda^{+\eta}$ while the ratio of the wavelength to the beam diameter scales as the inverse: 


$$
\left(\frac{\lambda}{D}\right)^{2} \sim \lambda^{-\eta}
$$

The parameter $\eta$ may assume any real value, but its preferred regime is the interval $[-2,0]$. Within this range, all laser and plasma parameters scale with wavelength from $\lambda^{-2}$ to $\lambda^{+2}$ while outside the interval at least one parameter will scale more strongly than the square and hence may become inconveniently large or small. We note that Schroeder's scaling is equivalent to $\eta=-2$ in our scheme. Table 1 summarizes the wavelength scaling of key laser, plasma, and accelerator parameters for the general wavelength scaling case, along with the four specific cases $\left(\eta=-2,-1,-\frac{2}{3}, 0\right)$. See the Appendix A for a list of symbols used.

Table 1. Net scaling of laser, plasma, and accelerator parameters with laser wavelength. The first column lists the various parameters (see the Appendix A for a list of symbols), the second lists the general wavelength scaling, and columns 3-6 show specific values of the relative scalings for a laser wavelength change from $0.8 \mu \mathrm{m}$ (Ti:S) to $1.9 \mu \mathrm{m}$ (Tm:YLF), a factor of $2.4 \times$. Unity values are in bold to highlight parameters fixed by the particular choices of the scaling parameter $\eta$.

\begin{tabular}{cccccc}
\hline Parameters & $\begin{array}{c}\text { General } \\
\text { Scaling }\end{array}$ & $\begin{array}{c}\text { Schroeder } \\
(\eta=-2)\end{array}$ & $\begin{array}{c}\text { Intermediate } \\
(\eta=-1)\end{array}$ & $\begin{array}{c}\text { Fixed Energy } \\
(\eta=-2 / 3)\end{array}$ & $\begin{array}{c}\text { Fixed Power and } \\
\text { Cycles }(\eta=0)\end{array}$ \\
\hline$a_{0}, E_{c m}, \Gamma$ & $\lambda^{0}$ & $\mathbf{1}$ & $\mathbf{1}$ & $\mathbf{1}$ & $\mathbf{1}$ \\
\hline$N_{\lambda}, f / \#$ & $\lambda^{+\eta / 2}$ & 0.4 & 0.6 & 0.7 & $\mathbf{1}$ \\
\hline$P, P_{c},\left(\omega / \omega_{p}\right)^{2}, \Delta W, N_{r}$ & $\lambda^{+\eta}$ & 0.2 & 0.4 & 0.6 & $\mathbf{1}$ \\
\hline$N_{\text {stages }}$ & $\lambda^{-\eta}$ & 5.6 & 2.4 & 1.8 & $\mathbf{1}$ \\
\hline$E, P_{\text {avg }}, E_{e}, L_{\text {stage }}$ & $\lambda^{+(1+3 \eta / 2)}$ & 0.2 & 0.6 & $\mathbf{1}$ & 2.4 \\
\hline$D, \tau, \lambda_{p}, N_{e}, L_{\text {tot }}, P_{\text {beam }}$, & $\lambda^{+(1+\eta / 2)}$ & $\mathbf{1}$ & 1.5 & 1.8 & 0.4 \\
\hline$P_{\text {wall }}$ & $\lambda^{-(1+\eta / 2)}$ & $\mathbf{1}$ & 0.6 & 0.6 & 0.2 \\
\hline$E_{z}, \omega_{p}, k_{p}$ & $\lambda^{-(2+\eta)}$ & $\mathbf{1}$ & 0.4 & 0.3 & 5.6 \\
\hline$n_{e}$ & $\lambda^{+(2+\eta)}$ & $\mathbf{1}$ & 2.4 & 3.2 & \\
\hline Luminosity, $\mathcal{L}$ & & & &
\end{tabular}

Choosing $\eta=-2$ (Schroeder) maintains the same end particle beam luminosity, plasma density, laser/wakefield diameter, total accelerator length, and requires the same total laser power for different choices of laser wavelength. However, it places extreme conditions on the number of stages $\left(\sim \lambda^{2}\right)$, significantly shortens acceleration length $\left(\sim \lambda^{-2}\right)$ per stage, and requires the laser to have the same pulsewidth but fewer cycles per pulse $\left(\sim \lambda^{-1}\right)$, and faster focal geometry with $\mathrm{f} / \# \sim \lambda^{-1}$. Therefore, to maintain the same accelerated electron energy and bunch charge with a laser with twice the wavelength, four times as many stages are needed, each being one quarter in length and providing only one quarter the energy per stage. The laser, while being the same total average power and pulse duration, would be at one quarter the pulse energy and peak power, twice the fractional bandwidth (half the number of cycles per pulse), and focused with half the focal ratio (twice the numerical aperture) to achieve the same focal spot diameter. This choice of scaling is the most stressing of the four discussed here upon the laser requirements and so most potentially at odds with laser scaling technology options.

At the opposite end of the scale, choosing $\eta=0$ yields a design with the identical number of stages as the original design. As the spot size and pulse duration scale linearly with wavelength in this scaling, $\eta=0$ scales congruently with the diffractive nature of light and hence is the least stressing on the laser. In addition, the $\mathrm{f} / \#$, cycles per pulse, laser peak power, fractional bandwidth, and accelerated electron energy are also constant under wavelength scaling. The necessary laser pulse energy, wallplug power, and acceleration length (both per stage and total) increases by $\lambda$, while the resonant plasma density drops by $\lambda^{-2}$, but the luminosity of the beam increases as $\lambda^{2}$. Although the wallplug power is increased linearly, the luminosity of the electron beam is increased by $\lambda^{2}$. Under the $\eta=0$ scaling, a $2 \times$ 
longer wavelength achieves the same electron energy using $2 \times$ more laser energy and pulse duration (same peak power), with $2 \times$ longer acceleration length, but produces $2 \times$ greater charge and bunch energy using the same number of stages each at one quarter the plasma density. Importantly, as this paper supports, some laser technologies can scale to higher power limits than others, and so operation at higher wallplug powers may be feasible at, for example, a longer wavelength (e.g., Thulium) and infeasible for a shorter choice of wavelength (e.g. TiS). The fact that luminosity grows by the square is, perhaps, a worthy trade for the technology choice.

Choosing $\eta=-2 / 3$ maintains the laser energy, average power, and stage length as the wavelength is varied. The number of stages grows sub-linearly as $\lambda^{2 / 3}$, as do the spot size, pulsewidth, number of accelerated electrons, and wall-plug power. Under the $\eta=-2 / 3$ scaling, a $2 \times$ longer wavelength laser achieves $0.6 \times$ the electron energy using $1.6 \times$ stages, each using the same laser energy, $1.6 \times$ spot size and pulse duration ( $0.6 \times$ peak power and $0.8 \times$ the number of cycles). The same acceleration length is needed, with $1.6 \times$ the charge and bunch energy produced, utilizing $0.4 \times$ the plasma density. While $1.6 \times$ wall plug power and total accelerator length would be required, the luminosity would increase by $2.5 \times$.

Choosing $\eta=-1$ results in intermediate scaling, in which none of the scaling factors (other than the by-design $a_{0}$ and electron energy $E_{c m}$ ) are unchanged with a choice of wavelength. However, all parameters are held to within the range of $\lambda^{ \pm 1}$ and hence none are scaling super-linearly. This choice of scaling perhaps best compromises among laser, plasma, and accelerator parameters. Under this scaling choice, a $2 \times$ longer wavelength laser achieves the same electron energy using two stages, each with $0.7 \times$ laser energy, $1.4 \times$ spot size and pulse duration $(0.5 \times$ peak power $), 0.7 \times$ acceleration length, and produces $0.7 \times$ charge and $1.4 \times$ bunch energy, at half the plasma density. Wall-plug power and total accelerator length would increase by $1.4 \times$, however, luminosity would increase by $2 \times$.

Importantly, top-level system trades must be looked at in evaluating the relative merits of different technology choices. For example, Figure 3 illustrates the laser peak/average power (at fixed, $10-\mathrm{kHz}$ rep-rate) trade space of equivalent LWFA operation. Starting from a single Ti:S design point corresponding to $100-\mathrm{J} / 100-\mathrm{fs}$ at $0.8-\mu \mathrm{m}$ wavelength, we show scaling lines for Tm:YLF $(1.9 \mu \mathrm{m})$ and $\mathrm{CO}_{2}(10 \mu \mathrm{m})$, but any choice of wavelength can be used and will be parallel to the lines shown. For Tm:YLF, the line of equivalent operating points spans from $240-\mathrm{J} / 240-\mathrm{fs} / 1-\mathrm{PW} / 24-\mathrm{kW}(\eta=0)$ to $18-\mathrm{J} / 100-\mathrm{fs} / 180-\mathrm{TW} / 1.8-\mathrm{kW}(\eta=-2)$. For $\mathrm{CO}_{2}$, this spans from 1.3-kJ/1.3ps/1-PW/125-kW $(\eta=0)$ to $0.6-\mathrm{J} / 100-\mathrm{fs} / 6.4-\mathrm{TW} / 64-\mathrm{W}(\eta=-2)$. These examples illustrate the large laser trade space that wavelength scaling opens up, and full system-level systems engineering studies need to be performed to understand where best to balance laser, plasma, and accelerator operating parameters.

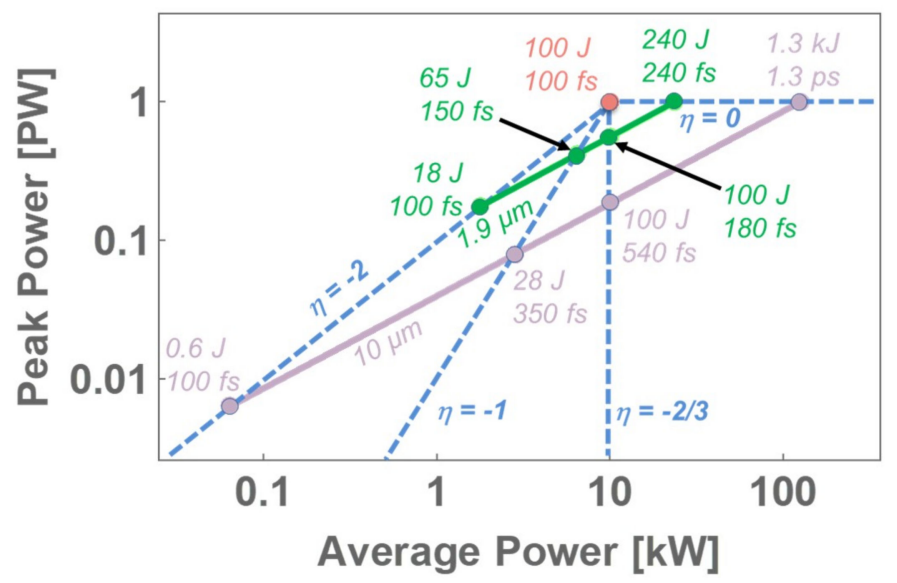

Figure 3. The $100 \mathrm{fs}, 100 \mathrm{~J}$ is the baseline design point at $0.8 \mu \mathrm{m}$. It should be noted that the number of accelerator stages is kept constant along the $\eta=0$ scaling line, the laser average power per stage is fixed along the $\eta=-2 / 3$ line, and the laser pulse duration is fixed along the $\eta=-2$ line. Note the considerable variation in laser parameters, all producing the same center-of-mass energy as the $0.8-\mu \mathrm{m}$ design point. 


\section{EuPRAXIA Design}

Case 1A in the EuPRAXIA D 1.2 report [12] calls for a $100 \mathrm{~J}, 100 \mathrm{fs}$ design, operating at $0.8 \mu \mathrm{m}$ at a repetition rate between $1 \mathrm{~Hz}$ and $100 \mathrm{~Hz}$. An MPE design with Tm:YLF as the gain material will operate at a wavelength near $1.9 \mu \mathrm{m}$. Following the wavelength scaling of the $\eta=0$ case in Table 1 , equivalent acceleration at $1.9 \mu \mathrm{m}$ may be achieved with a $240 \mathrm{fs}$ pulse with $240 \mathrm{~J}$ of energy. A repetition rate of $100 \mathrm{~Hz}$ was selected because a repetition rate higher than the inverse lifetime of the gain material ( $\sim 67 \mathrm{~Hz}$ for Tm:YLF) is necessary to operate an amplifier in the MPE regime. A second design point, operating at $200 \mathrm{~Hz}$, was considered to illustrate the advantages of going to higher repetition rates in MPE amplifiers.

The physical architecture of the amplifier is similar to that used in SHARC and BAT designs. The gain medium comprises gas-cooled slabs $[15,16]$ arranged into two main amplifier heads. The slabs are anti-reflection-coated for normal incidence light at both the pump and laser wavelengths. Each head is diode-pumped on both ends through dichroic mirrors. The extraction beam is relay imaged [17] between passes. A spatial filter is placed in between the two extraction heads [18]. The extraction beam is angularly multiplexed through the heads. Both 4-pass and 8-pass architectures were considered. An out-of-plane compressor [19] is used to compress the pulse.

Four individually optimized designs, sharing the same architecture described in the previous paragraph, were created: 4-pass and 8-pass versions of the $100 \mathrm{~Hz}$ and $200 \mathrm{~Hz}$ design points. The procedure discussed in Reference [20] was used to optimize each of the four designs, with one change: instead of having two equal-area heads, the aperture sizes of both heads are scaled so that their output fluences on the last pass are at the operating fluence limit (a value set below the laser damage fluence). This change increases the fluence in the second head and thus enables more efficient extraction.

Table 2 shows key performance metrics of various MPE EuPRAXIA designs. For every design, the input energy to the amplifier is $1 \mathrm{~J}$, and the input spectrum is sculpted to pre-compensate for distortion caused by saturation of the amplifier and the gain spectrum of Tm:YLF. The spectral output of the amplifier is a $41 \mathrm{~nm}$ FWHM $8^{\text {th }}$ order super-Gaussian, capable of supporting $240 \mathrm{fs}$ pulses when compressed. A sample of the sculpted input and resultant output of the amplifier is shown in Figure 4 . The energy output of the amplifier is $282 \mathrm{~J}$, which is reduced to $240 \mathrm{~J}$ after the compression. The B-integral is only $0.1 \mathrm{rad}$ for 4-pass designs and $0.2 \mathrm{rad}$ for 8-pass designs because of the relatively low extraction fluence possible with MPE extraction.

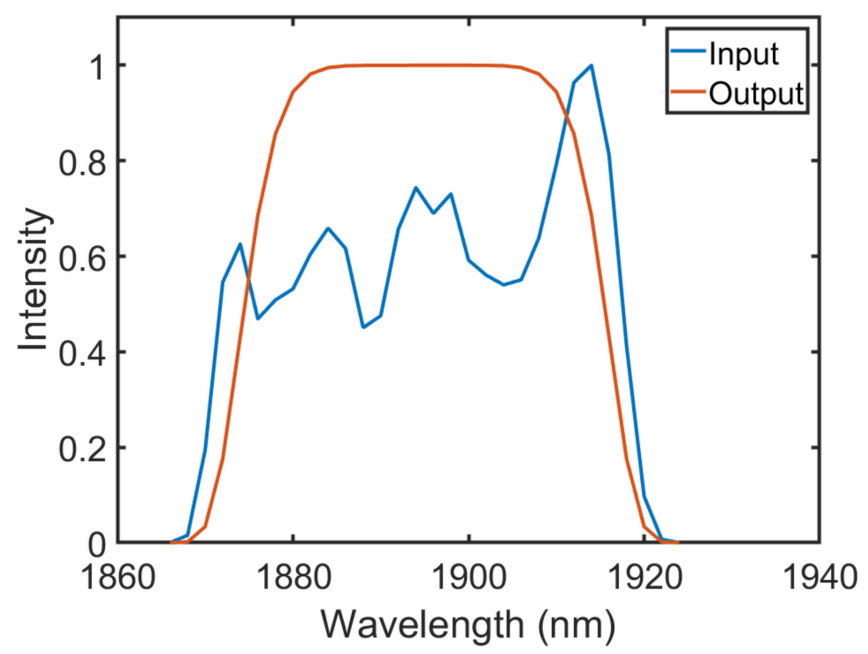

Figure 4. Simulated input and output spectral shapes of main amplifier when operating at $100 \mathrm{~Hz}$. The output is a $41 \mathrm{~nm}$ FWHM 8-th order super-Gaussian. The shape of the input spectrum was adjusted (sculpted) so that the resultant output shape would be an 8-th order super-Gaussian. 
A key quantity to consider in MPE amplifiers is the storage efficiency, defined as the fraction of upper state population which does not decay between pulses. A lower repetition rate means a longer time between pulses, and, consequently, a greater fraction of population lost to fluorescence decay. MPE amplifiers operate most efficiently when their repetition rate is much greater than the inverse lifetime of the gain material. At a repetition rate of $100 \mathrm{~Hz}$, the storage efficiency for a Tm:YLF $\left(\tau_{\text {storage }}=15 \mathrm{~ms}\right)$ amplifier is $51 \%$, but, at $200 \mathrm{~Hz}$, the storage efficiency increases to $72 \%$. Two $200 \mathrm{~Hz}$ designs (4-pass and 8-pass) producing the same output energy as the corresponding $100 \mathrm{~Hz}$ designs are summarized in the second and fourth columns of Table 2. The gain in efficiency in going to a higher repetition rate is such that twice the average laser output power can be obtained with only a $\sim 10 \%$ increase in optical pump power. The optical-to-optical efficiency continues to increase for amplifiers optimized at higher repetition rates. With MPE, it can be easier to create amplifiers optimized for higher repetition rates.

Even when each individual pulse only extracts a small fraction of the upper-state population, MPE amplifiers can still be operated efficiently. However, efficiency does increase with the extraction per pulse. The extraction per pulse increases with the extraction fluence, summed over all passes. Because EuPRAXIA designs are already operated near the damage fluence, the extraction efficiency cannot be increased by making the aperture smaller. Instead, a design with a greater number of passes can be used. As seen in the third and fourth columns of Table 2, the 8-pass designs are more efficient than the corresponding 4-pass designs. The optical-to-optical conversion efficiency of the $200 \mathrm{~Hz} 8$-pass design is over $14 \%$. The analysis performed did not consider complications which may present themselves from the extra passes, such as increased thermal beam distortion. A more detailed study is necessary to more accurately assess the benefits of the 8-pass design.

Table 2. Optimized performance of $100 \mathrm{~Hz}$ and $200 \mathrm{~Hz}$ Tm:YLF MPE designs.

\begin{tabular}{lcccc}
\hline \multicolumn{1}{c}{ Parameter } & $\begin{array}{c}\mathbf{1 0 0 ~} \mathbf{~ H z} \\
\mathbf{4} \text { Pass }\end{array}$ & $\begin{array}{c}\mathbf{2 0 0 ~} \mathbf{~ H z} \\
\mathbf{4} \text { Pass }\end{array}$ & $\begin{array}{c}\mathbf{1 0 0 ~} \mathbf{~ H z} \\
\mathbf{8 ~ P a s s}\end{array}$ & $\begin{array}{c}\mathbf{2 0 0} \mathbf{~ H z} \\
\mathbf{8} \text { Pass }\end{array}$ \\
\hline Seed Energy (J) & 1 & 1 & 1 & 1 \\
\hline Amplifier Output Energy (J) & 282 & 282 & 282 & 282 \\
\hline Compressed Energy (J) & 240 & 240 & 240 & 240 \\
\hline Pulse Duration (fs) & 240 & 240 & 240 & 240 \\
\hline Pump Power (kW) & 501 & 557 & 343 & 392 \\
\hline Wall-plug Power (kW) & 1206 & 1327 & 820 & 929 \\
\hline Optical-to-Optical Efficiency & 0.056 & 0.101 & 0.082 & 0.143 \\
\hline Sculpting Depth & 2.4 & 8 & 3.4 & 3.8 \\
\hline Stretched Pulse Duration (ns) & 3 & 3 & 3 & 3 \\
\hline B-integral (rad) & 0.10 & 0.10 & 0.16 & 0.15 \\
\hline
\end{tabular}

When the repetition rate of an MPE amplifier is much greater than the inverse lifetime of the gain material, the storage efficiency of the gain medium approaches unity. Extraction is then continuous from the material's point of view and only average power is important. Thus, with no physical changes to the amplifier, output pulse energy and repetition rate may be freely exchanged, provided that the average power of all inputs (seed and pump) remain constant. As previously noted, EuPRAXIA designs are operated near the damage fluence limit and they cannot be operated at lower repetition rate and higher energy than their initial design point. However, they may be operated at lower energy and higher repetition rate. Figure 5 shows the performance of various MPE EuPRAXIA designs as their repetition rates are increased. The six-pointed stars indicate the point at which each design was optimized. Figure 5a shows the energies which may be achieved at various repetition rates up to $10 \mathrm{kHz}$, and the amplifiers may be operated at even higher repetition rates if desired. The 
optical-to-optical efficiencies are plotted in Figure 5b. The slight drop in optical-to-optical efficiency with repetition rate is because the repetition rates of less than $200 \mathrm{~Hz}$ are insufficient to saturate the storage efficiency of Tm:YLF. As a result, the average power scaling is not perfectly obeyed. The flat optical-to-optical efficiency of the BAT baseline design above $1 \mathrm{kHz}$ illustrates more ideal repetition rate scaling of MPE designs.
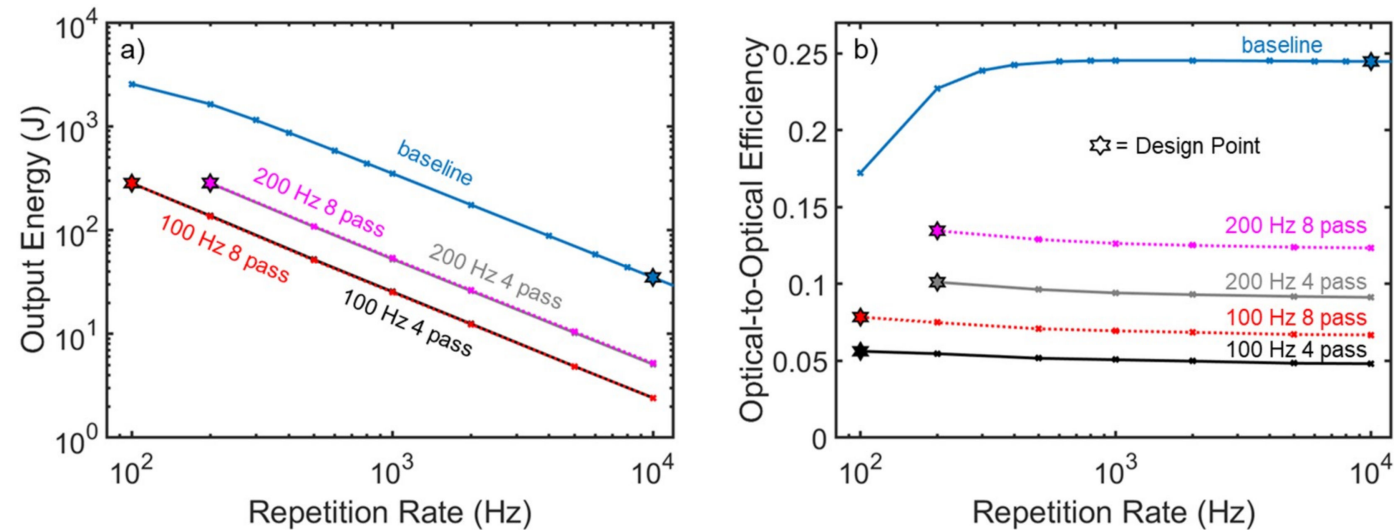

Figure 5. (a) Available output energy from EuPRAXIA amplifier designs as it is operated at different repetition rates when tuned at constant average power. The energy tuning curves for the 4-pass and 8-pass versions of the designs are nearly indistinguishable. (b) Optical-to-optical efficiency of designs as a function of repetition rate. The 'baseline' design is the $10 \mathrm{kHz}, 100 \mathrm{fs}, 30 \mathrm{~J}$ design described in [20].

Figure 6 shows a scatter plot of average output power versus wall-plug electrical power for the amplifiers discussed in this paper. Following the curves of constant wall-plug efficiency (upward-sloping lines on the plot), it is apparent that scaling the average output power of SPE designs while maintaining constant wall-plug efficiency will result in unacceptable wall-plug power requirements when average output powers reach the $100 \mathrm{~kW}$ regime-for example, the lamp-pumped LPSSLP architecture would require 10's of MW's of electrical power to produce 100-kW laser output. While SPE designs may provide a near term increase in average power, in the long term, higher efficiency designs will be required, exemplified by MPE Tm:YLF designs.

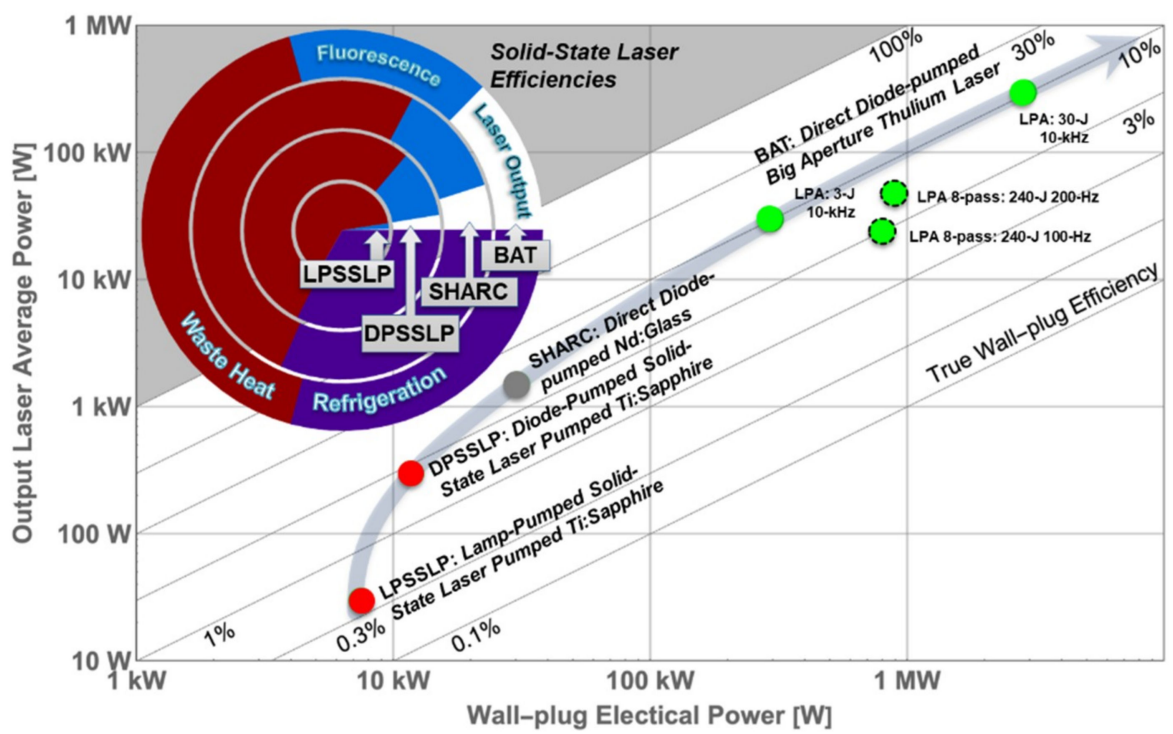

Figure 6. Wall-plug and average laser output power of various amplifier design points. The 8-pass $100 \mathrm{~Hz}$ and $200 \mathrm{~Hz}$ design points discussed in this section are outlined in black dashed lines. The $30 \mathrm{~J}$ $10 \mathrm{kHz}$ design is the baseline BAT design described in Reference [20], while the $3 \mathrm{~J} 10 \mathrm{kHz}$ design is its 
corresponding pump laser. The inset wheel chart shows fraction of wallplug energy moved to laser output, fluorescence, waste heat, and refrigeration. Colors of the dots indicate the material from which the amplifier is made, following the same convention as the key in Figure 1.

\section{Conclusions}

We have presented a new wavelength scaling of LWFA operation within which technology choices, such as drive laser architecture, can be evaluated in a top-level system engineering context. Importantly, the fact that some laser architectures have fundamental and/or practical limits to power scaling introduces trade-space constraints with broader implications, which demand deeper community exploration and evaluation.

Author Contributions: Conceptualization: C.S., A.E., A.B., B.R. and E.S.; Data Curation: B.R.; Formal Analysis: C.S.; Funding Acquisition: A.B., E.S., T.S., and C.H.; Investigation: C.S. and T.G.; Software: T.G.; Supervision: E.S., T.S., and C.H.; Validation: T.G.; Writing: C.S. and T.G.

Funding: This work was performed under the auspices of the U.S. Department of Energy by Lawrence Livermore National Laboratory under Contract DE-AC52-07NA27344 and was supported by the LLNL-LDRD Program under Project No. 17-ERD-033.

Conflicts of Interest: The authors declare no conflict of interest.

\section{Appendix A}

Table A1. Summary of Symbols.

\begin{tabular}{|c|c|c|c|}
\hline Variable & Meaning & Variable & Meaning \\
\hline$a_{0} \equiv q A / m_{e} c^{2}$ & $\begin{array}{l}\text { normalized vector potential of } \\
\text { laser field }\end{array}$ & $P$ & peak laser power \\
\hline$c$ & speed of light in vacuum & $P_{a v g}$ & Laser average power \\
\hline$D$ & $\begin{array}{l}\text { laser spot intensity FWHM } \\
\text { diameter }\end{array}$ & $P_{\text {beam }}$ & Electron beam average power \\
\hline E & energy of laser pulse & $P_{c} \equiv 2 P_{r}\left(\frac{\omega}{\omega_{p}}\right)^{2}$ & $\begin{array}{l}\text { critical laser power for laser } \\
\text { self-focusing }\end{array}$ \\
\hline$E_{c m}=2 \gamma m_{e} c^{2}$ & center of mass collision energy & $P_{r} \equiv \frac{m_{e} c^{2}}{r_{e} / c}$ & $\begin{array}{l}\text { Characteristic relativistic } \\
\text { power. }\end{array}$ \\
\hline$E_{e}$ & energy of electron bunch & $P_{\text {wall }}$ & $\begin{array}{l}\text { wallplug power of entire } \\
\text { accelerator }\end{array}$ \\
\hline$E_{z}$ & $\begin{array}{l}\text { longitudinal electric field in } \\
\text { wakefield stage }\end{array}$ & $q$ & electron charge \\
\hline$f$ & $\begin{array}{l}\text { frequency of laser pulses and } \\
\text { collider collision frequency }\end{array}$ & $r_{e}$ & classical electron radius \\
\hline$f / \#$ & f-number of laser focus & $v$ & velocity of electrons \\
\hline$k_{p} \equiv \omega_{p} / c$ & plasma wavenumber & $\gamma \equiv\left(1-\left(\frac{v}{c}\right)^{2}\right)^{-1 / 2}$ & relativistic Lorentz factor \\
\hline $\mathcal{L}$ & luminosity of colliding beams & $\Gamma \equiv \frac{E_{\text {beam }}}{E}$ & $\begin{array}{l}\text { efficiency of laser energy to } \\
\text { electron bunch energy }\end{array}$ \\
\hline$L_{\text {stage }}$ & $\begin{array}{l}\text { acceleration length of a single } \\
\text { wakefield stage }\end{array}$ & $\Delta W$ & $\begin{array}{l}\text { energy gain of individual } \\
\text { electron in one stage of } \\
\text { accelerator }\end{array}$ \\
\hline$L_{t o t}$ & $\begin{array}{l}\text { total length of entire acceleration } \\
\text { chain (minus inter-stage regions) }\end{array}$ & $\eta$ & $\begin{array}{l}\text { wavelength scaling parameter } \\
\text { of LWFA design point }\end{array}$ \\
\hline$n_{e}$ & electron density of plasma & $\lambda_{p}$ & plasma wavelength \\
\hline
\end{tabular}


Table A1. Cont.

\begin{tabular}{llll}
\hline \multicolumn{1}{c}{ Variable } & \multicolumn{1}{c}{ Meaning } & Variable & \multicolumn{1}{c}{ Meaning } \\
\hline$N_{e}$ & $\begin{array}{l}\text { number of accelerated electrons } \\
\text { per bunch }\end{array}$ & $\sigma$ & $\begin{array}{l}\text { RMS beam size at interaction } \\
\text { point }\end{array}$ \\
\hline$N_{r} \equiv P / P_{r}$ & $\begin{array}{l}\text { Dimensionless laser power, scaled } \\
\text { by characteristic relativistic power }\end{array}$ & $\tau$ & duration of laser pulse \\
\hline$N_{\text {stages }}$ & number of LPWA stages & $\omega$ & laser frequency \\
\hline$N_{\lambda} \equiv \tau v=\frac{E c / \lambda}{P}$ & Number of laser cycles per pulse & $\omega_{p}$ & plasma frequency \\
\hline
\end{tabular}

\section{References}

1. Tajima, T.; Dawson, J.M. Laser Electron Accelerator. Phys. Rev. Lett. 1979, 43, 267. [CrossRef]

2. Strickland, D.; Mourou, G. Compression of Amplified Chirped Optical Pulses. Opt. Comm. 1985, 56, 219. [CrossRef]

3. Hamster, H.; Sullivan, A.; Gordon, S.; Falcone, R.W. Short-Pulse Terahertz Radiation From High-Intensity-Laser-Produced Plasmas. Phys. Rev. E 1994, 49, 671. [CrossRef] [PubMed]

4. Siders, C.W.; LeBlanc, S.P.; Fisher, D.; Tajima, T.; Downer, M.C.; Babine, A.; Stepanov, A.; Sergeev, A. Laser Wakefield Excitation and Measurement by Femtosecond Longitudinal Interferometry. Phys. Rev. Lett. 1996, 76, 3570. [CrossRef] [PubMed]

5. Marques, J.R.; Geindre, J.P.; Amiranoff, F.; Audebert, P.; Gauthier, J.C.; Antonetti, A.; Grillon, G. Temporal and Spatial Measurements of the Electron Density Perturbation Produced in the Wake of an Ultrashort Laser Pulse. Phys. Rev. Lett. 1996, 76, 3566. [CrossRef] [PubMed]

6. Modena, A.; Najmudln, Z.; Dangor, A.E.; Clayton, C.E.; Marsh, K.A.; Joshi, C.; Malka, V.; Darrow, C.B.; Danson, C.; Neely, D.; et al. Electron acceleration from the breaking of relativistic plasma waves. Lett. Nat. 1995, 377, 606. [CrossRef]

7. Ting, A.; Moore, C.I.; Krushelnick, K.; Manka, C.; Esarey, E.; Sprangle, P.; Hubbard, R.; Burris, H.R.; Fischer, R.; Baine, M. Plasma Wakefield Generation and Electron Acceleration in a Self-Modulated Laser Wakefield Accelerator Experiment. Phys. Plasma. 1998, 4, 1889. [CrossRef]

8. Esarey, E.; Schroeder, C.B.; Leemans, W.P. Physics of laser-driven plasma-based electron accelerators. Rev. Mod. Phys. 2009, 81, 1229. [CrossRef]

9. Haefner, C.L.; Bayramian, A.; Betts, S.; Bopp, R.; Buck, S.; Cupal, J.; Drouin, M.; Erlandson, A.; Horáček, J.; Horner, J.; et al. High average power, diode pumped petawatt laser systems: a new generation of lasers enabling precision science and commercial applications. SPIE Proc. 2017, 10241, 1024102-1024104.

10. Schroeder, C.B.; Esarey, E.; Geddes, C.G.R.; Benedetti, C.; Leemans, W.P. Physics considerations for laser-plasma linear colliders. Phys. Rev. Spec. Top. 2010, 13, 101301. [CrossRef]

11. U.S. DOE Office of Science. Advanced Accelerator Development Strategy Report. In Proceedings of the DOE Advanced Accelerator Concepts Research Roadmap Workshop, Berkeley, CA, USA, 2-3 February 2016.

12. Walker, P.A. Eupraxia Deliverable Report D 1.2 Report defining preliminary study concept 2016. Available online: https:/indico.cern.ch/event/555407/contributions/2263620/attachments/1333501/2004985/EuPRAXIA_ deliverable_1.2_V0.1.pdf (accessed on 19 August 2019).

13. AWAKE Advanced Wakefield Experiment. Available online: http://awake.web.cern.ch/awake/ (accessed on 22 July 2019).

14. Blumfeld, I.; Clayton, C.E.; Decker, F.-J.; Hogan, M.J.; Huang, C.; Ischebeck, R.; Iverson, R.; Joshi, C.; Katsouleas, T.; Kirby, N.; et al. Energy doubling of $42 \mathrm{GeV}$ electrons in a metre-scale plasma wakefield accelerator. Nat. Lett. 2007, 445, 741. [CrossRef] [PubMed]

15. Emmett, J.L.; Krupke, W.F.; Sooy, W.R. The Potential of High-Average-Power Solid State Lasers; Livermore, CA, USA: Lawrence Livermore National Lab, 1984; No. UCRL-5371.

16. Bayramian, A.; Armstrong, P.; Ault, E.; Beach, R.; Bibeau, C.; Caird, J.; Campbell, R.; Chai, B.; Dawson, J.; Ebbers, C.; et al. The Mercury Project: A High Average Power, Gas-cooled Laser for Inertial Fusion Energy Development. Fusion Sci. Technol. 2007, 52, 383. [CrossRef] 
17. Hunt, J.T.; Glaze, J.A.; Simmons, W.W.; Renard, P.A. Suppression of self-focusing through low-pass spatial filtering and relay imaging. App. Opt. 1978, 17, 2053. [CrossRef] [PubMed]

18. Erlandson, A.; Bayramian, A. Spatial filters for high power lasers. U.S. Patent No.8,902,497, 2012.

19. Alessi, D.A.; Sistrunk, E.; Nguyen, H.T.; Rosso, P.A.; Spinka, T.; Aasen, M.D.; Herriot, S.; Britten, J.A.; Haefner, C. A Compressor for High Average Power Ultrafast Laser Pulses with High Energies. In Proceedings of the Conference on Lasers and Electro-Optics 2017, San Jose, CA, USA, 14-19 May 2017.

20. Galvin, T.C.; Bayramian, A.; Chesnut, K.D.; Erlandson, A.; Siders, C.W.; Spinka, T.; Haefner, C. Scaling of petawatt-class lasers to multi-kHz repetition rates. Proc. SPIE 2019, 11033, 11033031-11033038.

(C) 2019 by the authors. Licensee MDPI, Basel, Switzerland. This article is an open access article distributed under the terms and conditions of the Creative Commons Attribution (CC BY) license (http://creativecommons.org/licenses/by/4.0/). 